Article

\title{
Synthesis and Biological Properties of Novel Triazole-Thiol and Thiadiazole Derivatives of the 1,2,4-Triazole-3(5)-one Class
}

\author{
Esra Düğdü *, Yasemin Ünver, Dilek Ünlüer and Kemal Sancak \\ Department of Chemistry, Karadeniz Technical University, Trabzon 61080, Turkey \\ * Author to whom correspondence should be addressed; E-Mail: esradugdu@gmail.com.
}

Received: 28 November 2013; in revised form: 7 January 2014 / Accepted: 8 January 2014 /

Published: 19 February 2014

\begin{abstract}
Butane-1,4-diyl/hexane-1,6-diyl)bis(3-methyl-5-oxo-4,5-dihydro-1,2,4triazole-4,1-diyl)) diacetohydrazides 3a,b were obtained via the formation of diethyl 2,2'-(4,4'(butane-1,4-diyl/hexane-1,6-diyl)bis(3-methyl-5-oxo-4,5-dihydro-1,2,4-triazole-4,1diyl))diacetates 2a,b, obtained starting from di-[3(methyl-2-yl-methyl)-4,5-dihydro- $1 H$ $[1,2,4]$-triazole-5-one-4yl]- $n$-alkanes $\mathbf{1 a , b}$ in two steps. The synthesis of the compounds $\mathbf{7 a}, \mathbf{b}-\mathbf{9 a}, \mathbf{b}$ incorporating the 1,3,4-thiadiazole, and 10a,b-11a,b with a 1,2,4-triazole-thiol nucleus as the second heterocycle, was performed by the acidic or basic treatment of compounds $\mathbf{4 a , b - 6 a , b}$ which were obtained from the reaction of $\mathbf{3 a}, \mathbf{b}$ with several isothiocyanates. Newly synthesized compounds were screened for antimicrobial activities and their antioxidant properties by the 1,1-diphenyl-2-picryl hydrazyl (DPPH) radical scavenging method. Compounds $4 \mathbf{a}, \mathbf{b}, \mathbf{5 a}, \mathbf{b}$, and $\mathbf{6 a}, \mathbf{b}$ were found to possess good antioxidant properties. Almost all compounds have significant antimicrobial activities.
\end{abstract}

Keywords: 1,2,4-triazole-5-one; 1,2,4-triazole-thiol; 1,3,4-thiadiazole; antioxidant and antimicrobial activities

\section{Introduction}

Derivatives of 1,3,4-thiadiazoles and 1,2,4-triazole are known to exhibit anti-inflammatory, antiviral, analgesic, antimicrobial, anticonvulsant and antidepressant activity, the latter being usually explored by the forced swim test [1-10]. Among the pharmacological profiles of 1,3,4-thiadiazoles and 1,2,4-triazoles, their antimicrobial, anticonvulsant and antidepressant properties seem to be the best documented. Triazoles, in particular, substituted-1,2,4-triazoles and the open-chain thiosemicarbazide 
counterparts of 1,2,4-triazole, are among the various heterocycles that have received the most attention during the last two decades as potential antimicrobial agents [11]. Substitutions including thio-, alkylthioand alkenylthio-derivatives have been carried out primarily at the 3-position of the 1,2,4-triazole ring, to afford potential antimicrobial agents that will overcome the abovementioned resistance problems.

Heterocycles containing a 1,2,4-triazole or 1,3,4-thiadiazole moiety, and the compounds consisting of 1,2,4-triazole and 1,3,4-thiadiazole condensed nucleus systems constitute a class of compounds possessing a wide spectrum of biological activities such as anti-inflammatory, antiviral and antimicrobial and antitumoral properties [12-18]. It was reported that more efficacious antibacterial compounds can be designed by joining two or more biologically active heterocyclic systems together in a single molecular framework. Keeping this observation in mind, this paper has presented the synthesis of new triazole thiadiazole derivatives incorporating different pharmacophores as hybrid molecules possessing antioxidant and antimicrobial activities [19].

\section{Results and Discussion}

The synthesis of the intermediate and target compounds was performed according to the reactions outlined in Scheme 1. The starting compounds $\mathbf{1 a}, \mathbf{b}$ were prepared following a previously reported literature procedure [20]. The reaction of compounds $\mathbf{1 a , b}$ with ethyl bromoacetate in the presence of sodium ethoxide produced diethyl 2,2'-(4,4'(butane-1,4-diyl/hexane-1,6-diyl)bis(3-methyl-5-oxo-4,5dihydro-1,2,4-triazole-4,1-diyl))diacetates 2a,b. The ethoxy group on compounds 2a,b is an good leaving group for further nucleophilic substitution, thus reactions of $\mathbf{2 a , b}$ with hydrazine hydrate converted these esters into the corresponding 2,2'-(4,4'(butane-1,4-diyl/hexane-1,6-diyl)bis(3-methyl5-oxo-4,5-dihydro-1,2,4-triazole-4,1diyl))diacetohydrazides derivatives $\mathbf{3 a , b}$ which were employed as key intermediates for synthesis of the target compounds.

Analytical and spectroscopic data of compounds $\mathbf{2 a}, \mathbf{b}$ confirmed this reaction by the additional signals derived from the $-\mathrm{CH}_{2} \mathrm{CO}_{2}$ Et group at the expected chemical shift values. Moreover, compounds $2 \mathbf{a}, \mathbf{b}$ gave a stable $\mathrm{M}+1$ ion peak. The ${ }^{1} \mathrm{H}-\mathrm{NMR}$ spectra of compounds 3a,b displayed no signals belonging to the $-\mathrm{OCH}_{2} \mathrm{CH}_{3}$ group; instead, new signals derived from the hydrazide structure

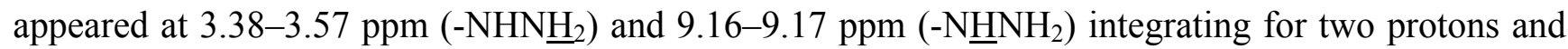
one proton, respectively $\left(\mathrm{D}_{2} \mathrm{O}\right.$ exchange). Furthermore, compounds 3a,b gave relatively stable $\mathrm{M}+1$ ion peaks.

N-(4-Halo/methyl- phenyl)-2-(2-(4-(4-(1-(2-(2-(4-halo/methyl- phenylcarbamothioyl)hydrazinyl)-2oxoethyl)-3-methyl-5-oxo-1 $H$-1,2,4-triazole-4(5H)-yl)alkyl)-3-methyl-5-oxo-4,5-dihydro-1,2,4-triazole1-yl)acetyl)hydrazinecarbothioamides $\mathbf{4 a}, \mathbf{b}-\mathbf{6 a}, \mathbf{b}$ were obtained by the reaction of compounds 3a,b with 4-fluorophenylisothiocyanate (4), 4-bromophenylisothiocyanate (5), or p-tolylisothiocyanate (6). The reaction was carried out at reflux temperature in ethanol and afforded the desired thiosemicarbazide derivatives, which were the starting materials for further cyclizations. The IR spectrum of compounds 4a,b-6a,b displayed a broad signal at $3245 \mathrm{~cm}^{-1}$ due to three $\mathrm{NH}$ absorptions. In the ${ }^{1} \mathrm{H}-\mathrm{NMR}$ spectra of compounds 4a,b-6a,b, these groups were observed at 9.56, 9.74, $10.24 \mathrm{ppm}$. Different from compound 3a,b, the ${ }^{1} \mathrm{H}$ - and ${ }^{13} \mathrm{C}-\mathrm{NMR}$ spectra of compounds $\mathbf{4 a}, \mathbf{b}-\mathbf{6 a}, \mathbf{b}$ exhibited additional signals due to thiosemicarbazide moiety at the expected chemical shift values. In addition, compounds $\mathbf{4 a}, \mathbf{b}-\mathbf{6 a}, \mathbf{b}$ gave relatively stable molecular ion peaks in the corresponding mass spectra. 
Scheme 1. Synthetic pathway for the preparation of compounds 2-11.
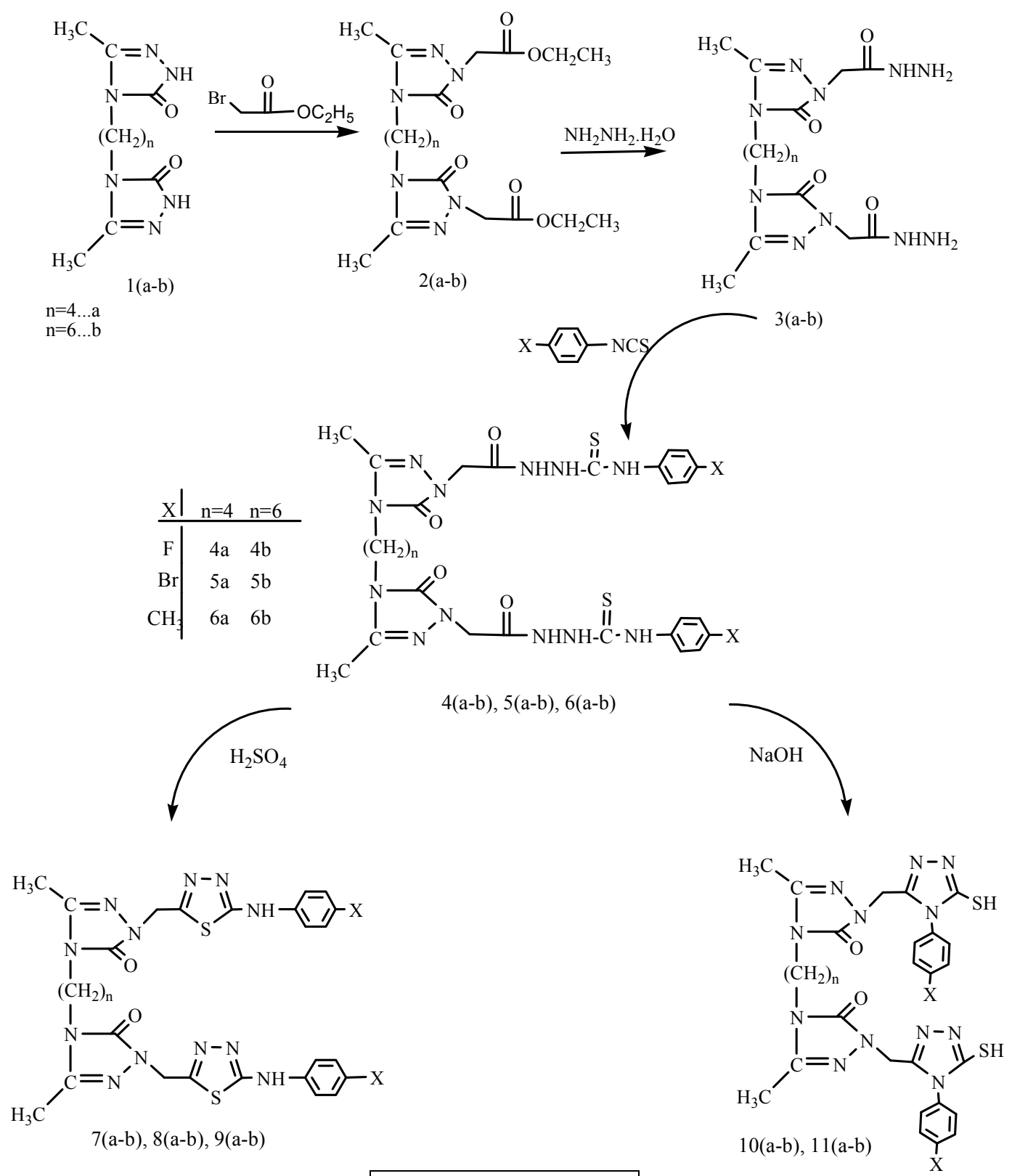

\begin{tabular}{|ccc|}
\hline $\mathrm{X}$ & $\mathrm{n}=4$ & $\mathrm{n}=6$ \\
\hline $\mathrm{F}$ & $\mathbf{7 a}, \mathbf{1 0 a}$ & $\mathbf{7 b}, \mathbf{1 0 b}$ \\
$\mathrm{Br}$ & $\mathbf{8 a}, \mathbf{1 1 a}$ & $\mathbf{8 b}, \mathbf{1 1 b}$ \\
$\mathrm{CH}_{3}$ & $\mathbf{9 a}$ & $\mathbf{9 b}$ \\
\hline
\end{tabular}

The treatment of compounds $\mathbf{4 a}, \mathbf{b}-\mathbf{6} \mathbf{a}, \mathbf{b}$ with cold concentrated sulfuric acid caused the conversion of the thiosemicarbazide structures into 1,3,4-thiadiazole rings; thus, 4,4'-( butane-1,4-diyl/hexane-1,6diyl)bis(2-((5-(4-halogen/methylphenylamino)1,3,4-thiadiazole-2-yl)methyl)-5-methyl-2H-1,2,4-triazole$3(4 H)$-ones 7a $, \mathbf{b}-9 \mathbf{a}, \mathbf{b}$ were obtained. On the other hand, the cyclization of the same intermediates, 4a,b-6a, $\mathbf{b}$ in the presence of $2 \mathrm{~N} \mathrm{NaOH}$ produced 4,4'-(butane-1,4-diyl/hexane-1,6-diyl)bis(2-((4-(4halogen/methylphenyl)-5-mercapto-4H-1,2,4-triazole-3-yl)methyl)-5-methyl-2H-1,2,4-triazole-3(4H)ones 10a,b and 11a,b. In the IR spectra of compounds $7 \mathbf{a}, \mathbf{b}-\mathbf{9 a}, \mathbf{b}$ the NH stretching band appeared at $3285-3198 \mathrm{~cm}^{-1}$. The NH proton resonated at about $10.20-10.27 \mathrm{ppm}$ in the ${ }^{1} \mathrm{H}-\mathrm{NMR}$ spectra. The IR 
spectra of compounds 10a,b and 11a,b displayed SH stretching bands at 2962-2988 $\mathrm{cm}^{-1}$. Moreover, in the ${ }^{1} \mathrm{H}-\mathrm{NMR}$ spectra of compounds $\mathbf{1 0 a}, \mathbf{b}$ and $\mathbf{1 1 a}, \mathbf{b}$, additional signals due to the $\mathrm{SH}$ group were observed at 13.88-13.99 ppm ( $\mathrm{D}_{2} \mathrm{O}$ exchangeable), while the $\mathrm{NH}$ signals disappeared.

The newly synthesized compounds were screened for antioxidant properties by radical scavenging methods such as the 1,1-diphenyl-2-picryl hydrazyl (DPPH) assay. Compounds 4a,b-6a,b possessing triazole-thiosemicarbazides have better antioxidant activity than $\mathbf{5 a}, \mathbf{b}$. While compounds $\mathbf{4 a}, \mathbf{b}$ and $\mathbf{6 a}, \mathbf{b}$ have fluorine and methyl group substituents on the phenyl rings, compounds $\mathbf{5 a}, \mathbf{b}$ have bromine substituents. Thiosemicarbazide derivatives $\mathbf{4 a}, \mathbf{b}-\mathbf{6 a}, \mathbf{b}$ also showed antibacterial activity against microorganisms. The 1,2,4-triazole nucleus is one of the active components present in many standard drugs and it is known to increase the pharmacological activity of the corresponding molecules. As an biological group, thiosemicarbazide groups in the triazole compounds should be considered for the synthesis of lead compounds in search of antioxidant and antimicrobial activity. All of the synthesized compounds were tested for antimicrobial activity. The antimicrobial screening suggests that among the newly synthesized compounds, 2a, 3a, 4a,b-11a,b exhibited moderate activity against some of the tested microorganisms.

\section{Experimental}

\subsection{General Information}

Melting points were measured on an electrothermal apparatus and are uncorrected. ${ }^{1} \mathrm{H}-\mathrm{NMR}$ and ${ }^{13} \mathrm{C}-\mathrm{NMR}$ spectra were recorded on a Varian XL-200 NMR spectrophotometer (Palo Alto, CA, USA) in DMSO- $d_{6}$. IR spectra were recorded on a Perkin-Elmer Spectrum one FT-IR spectrometer (Waltham, MA, USA) in $\mathrm{KBr}$ pellets. The MS spectra were measured with a Micromass Quattro LC/ULTIMA LC-MS/MS spectrometer (Waters, Milford, MA, USA) with EtOH as solvent. The experiment was performed in the positive ion mode. Elemental analyses were performed on a Hewlett-Packard $185 \mathrm{CHN}$ analyzer. All the chemicals were obtained from Fluka Chemie AG (Buchs, Switzerland).

\subsection{General Method for the Synthesis of Compounds 2}

The corresponding compound $1(0.01 \mathrm{~mol})$ was refluxed with an equivalent amount of sodium in absolute ethanol for $2 \mathrm{~h}$. Then, ethyl bromoacetate $(0.01 \mathrm{~mol})$ was added and the mixture was refluxed for an additional $5 \mathrm{~h}$. After evaporation of the solvent under reduced pressure at $35-40{ }^{\circ} \mathrm{C}$, a solid appeared. This was recrystallized from 1:1 ethanol/water to afford the desired product.

Diethyl 2,2'-(4,4'(butane-1,4-diyl)bis(3-methyl-5-oxo-4,5-dihydro-1,2,4-triazole-4,1-diyl))diacetate (2a). Yield 78\%, m.p. $150-151{ }^{\circ} \mathrm{C}$. IR (v, $\left.\mathrm{cm}^{-1}\right)$ : 1,755 (ester $\left.\mathrm{C}=\mathrm{O}\right), 1,698$ (triazole $\left.\mathrm{C}=\mathrm{O}\right), 1,638(\mathrm{C}=\mathrm{N})$, 1,212 (C-O); Anal. Calcd (\%) for $\mathrm{C}_{18} \mathrm{H}_{28} \mathrm{~N}_{6} \mathrm{O}_{6}$ : C, 50.93; H, 6.65; N, 19.80. Found: C, 50.98; H, 6.68; $\mathrm{N}, 19.85 ;{ }^{1} \mathrm{H}-\mathrm{NMR}(\delta \mathrm{ppm}): 1.14\left(6 \mathrm{H}, \mathrm{t}, J=7.0 \mathrm{~Hz}, 2 \mathrm{OCH}_{2} \mathrm{CH}_{3}\right), 1.52-1.59\left(4 \mathrm{H}, \mathrm{bs}, 2 \mathrm{NCH}_{2} \underline{\mathrm{H}}_{2}\right)$, $2.16\left(6 \mathrm{H}, \mathrm{s}, 2 \mathrm{CH}_{3}\right), 3.57\left(4 \mathrm{H}, \mathrm{t}, 2 \mathrm{NCH}_{2}\right), 4.08\left(4 \mathrm{H}, \mathrm{q}, J=7.0 \mathrm{~Hz}, 2 \mathrm{OC}_{2} \mathrm{CH}_{3}\right), 4.45(4 \mathrm{H}, \mathrm{s}, 2$ $\left.\mathrm{NCH}_{2} \mathrm{C}=\mathrm{O}\right) ;{ }^{13} \mathrm{C}-\mathrm{NMR}(\delta \mathrm{ppm}): 13.01\left(2 \mathrm{CH}_{3}\right), 19.94\left(2 \mathrm{OCH}_{2} \mathrm{CH}_{3}\right), 26.45\left(2 \mathrm{NCH}_{2} \underline{\mathrm{C}}_{2}\right), 43.79$ (2 
$\left.\mathrm{NCH}_{2}\right), 49.25\left(2 \mathrm{NCH}_{2} \mathrm{C}=\mathrm{O}\right), 62.11\left(2 \mathrm{OCH}_{2}\right), 147.96(2 \mathrm{C}=\mathrm{N}), 159.71(2 \mathrm{C}=\mathrm{O}), 169.74(2 \mathrm{C}=\mathrm{O}$ hydrazide $)$; MS (ESI): $m / z(\%) 425.21(\mathrm{M}+1)$.

Diethyl 2,2'-(4,4'(hexane-1,6-diyl)bis(3-methyl-5-oxo-4,5-dihydro-1,2,4-triazole-4,1-diyl))diacetate (2b). Yield 80\%, m.p. $142-143{ }^{\circ} \mathrm{C}$. IR (v, $\left.\mathrm{cm}^{-1}\right)$ : 1748 (ester C=O), 1698 (triazole C=O), $1618(\mathrm{C}=\mathrm{N}), 1208$ (C-O); Anal. Calcd (\%) for $\mathrm{C}_{20} \mathrm{H}_{32} \mathrm{~N}_{6} \mathrm{O}_{6}$ : C, 53.09; H, 7.13; N, 18.57. Found: C, 53.14; H, 7.17; N, 18.53; ${ }^{1} \mathrm{H}-\mathrm{NMR}\left(\mathrm{DMSO}-d_{6}, \delta \mathrm{ppm}\right):{ }^{1} \mathrm{H}-\mathrm{NMR}(\delta \mathrm{ppm}): 1.20\left(6 \mathrm{H}, \mathrm{bs}, 2 \mathrm{OCH}_{2} \mathrm{C}_{3}\right), 1.52(4 \mathrm{H}, \mathrm{bs}$, $\left.2 \mathrm{NCH}_{2} \mathrm{C}_{2}\right), 2.16\left(6 \mathrm{H}, \mathrm{s}, 2 \mathrm{CH}_{3}\right), 2.46\left(4 \mathrm{H}, \mathrm{bs}, 2 \mathrm{NCH}_{2} \mathrm{CH}_{2} \mathrm{C}_{2}\right), 4.09\left(4 \mathrm{H}, \mathrm{bs}, 2 \mathrm{NCH}_{2}\right), 4.11(4 \mathrm{H}, \mathrm{bs}$, $\left.2 \mathrm{OC}_{2} \mathrm{CH}_{3}\right), 4.46\left(4 \mathrm{H}, \mathrm{s}, 2 \mathrm{NCH}_{2} \mathrm{C}=\mathrm{O}\right) ;{ }^{13} \mathrm{C}-\mathrm{NMR}(\delta \mathrm{ppm}): 11.06\left(2 \mathrm{CH}_{3}\right), 20.01\left(2 \mathrm{OCH}_{2} \mathrm{CH}_{3}\right), 25.58$ $\left(2 \mathrm{NCH}_{2} \mathrm{CH}_{2} \underline{\mathrm{CH}} 2\right), 27.75\left(2 \mathrm{NCH}_{2} \underline{\mathrm{C}}_{2}\right), 43.66\left(2 \mathrm{NCH}_{2}\right), 49.26\left(2 \mathrm{NCH}_{2} \mathrm{C}=\mathrm{O}\right), 62.15\left(\mathrm{OCH}_{2}\right), 146.28$ $(2 \mathrm{C}=\mathrm{N}), 159.57(2 \mathrm{C}=\mathrm{O}), 169.72(2 \mathrm{C}=\mathrm{O}$ hydrazide); $\mathrm{MS}(\mathrm{ESI}): m / z(\%) 453.25(\mathrm{M}+1)$.

\subsection{General Method for the Synthesis of Compounds $\mathbf{3}$}

A solution of the corresponding compound $2(10 \mathrm{mmol})$ in $n$-butanol was refluxed with hydrazine hydrate $(25 \mathrm{mmol})$ for $4 \mathrm{~h}$. After cooling it to room temperature, a white solid appeared. This was recrystallized from 1:2 ethanol-water to obtain the desired compound.

2,2'-(4,4'(Butane-1,4-diyl)bis(3-methyl-5-oxo-4,5-dihydro-1,2,4-triazole-4,1-diyl)) diacetohydrazide (3a).

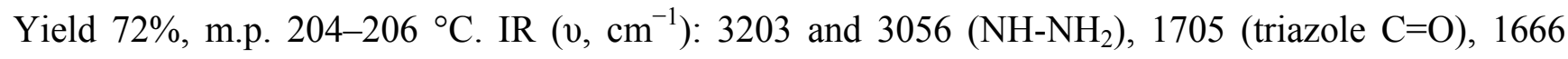
(hydrazide $\mathrm{C}=\mathrm{O}$ ); Anal. Calcd (\%) for $\mathrm{C}_{14} \mathrm{H}_{24} \mathrm{~N}_{10} \mathrm{O}_{4}$ : C, 42.42; $\mathrm{H}, 6.10 ; \mathrm{N}, 35.33$. Found: $\mathrm{C}, 42.45 ; \mathrm{H}$, 6.17; N, 35.38; ${ }^{1} \mathrm{H}-\mathrm{NMR}(\delta \mathrm{ppm}): 1.54\left(4 \mathrm{H}, \mathrm{bs}, 2 \mathrm{NCH}_{2} \mathrm{C}_{2}\right), 2.15\left(6 \mathrm{H}, \mathrm{s}, 2 \mathrm{CH}_{3}\right), 3.57-3.64(8 \mathrm{H}, \mathrm{bs}$, $\left.2 \mathrm{NCH}_{2}+2 \mathrm{NHNH}_{2}\right), 4.16\left(4 \mathrm{H}, \mathrm{s}, 2 \mathrm{NCH}_{2} \mathrm{C}=\mathrm{O}\right), 9.16\left(2 \mathrm{H}, \mathrm{s}, 2 \mathrm{NHNH}_{2}\right) ;{ }^{13} \mathrm{C}-\mathrm{NMR}(\delta \mathrm{ppm}): 11.09$ $\left(2 \mathrm{CH}_{3}\right), 26.24\left(2 \mathrm{NCH}_{2} \underline{\mathrm{CH}}_{2}\right), 41.37\left(2 \mathrm{NCH}_{2}\right), 46.76\left(2 \mathrm{NCH}_{2} \mathrm{C}=\mathrm{O}\right), 144.33(2 \mathrm{C}=\mathrm{N}), 154.38(2 \mathrm{C}=\mathrm{O})$, $170.12\left(2 \mathrm{C}=\mathrm{O}\right.$ hydrazide); $\mathrm{MS}(\mathrm{ESI}): \mathrm{m} / z(\%) 396.20\left(\mathrm{M}^{+1}\right)$.

2,2'-(4,4'(Hexane-1,6-diyl)bis(3-methyl-5-oxo-4,5-dihydro-1,2,4-triazole-4,1-diyl)) diacetohydrazide (3b).

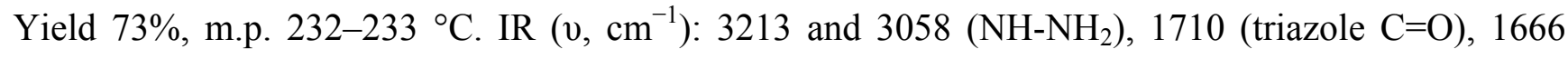
(hydrazide $\mathrm{C}=\mathrm{O}$ ); Anal. Calcd (\%) for $\mathrm{C}_{16} \mathrm{H}_{28} \mathrm{~N}_{10} \mathrm{O}_{4}$ : C, 45.27; $\mathrm{H}, 6.65 ; \mathrm{N}, 33.00$. Found: $\mathrm{C}$, 45.33; $\mathrm{H}$, 6.72; N, 33.05; ${ }^{1} \mathrm{H}-\mathrm{NMR}(\delta \mathrm{ppm}): 1.26\left(4 \mathrm{H}, \mathrm{bs}, 2 \mathrm{NCH}_{2} \mathrm{CH}_{2} \mathrm{CH}_{2}\right), 2.13\left(6 \mathrm{H}, \mathrm{s}, 2 \mathrm{CH}_{3}\right), 3.38-3.51(8 \mathrm{H}$, bs, $\left.2 \mathrm{NCH}_{2}+2 \mathrm{NHNH}_{2}\right), 4.17\left(4 \mathrm{H}, \mathrm{s}, 2 \mathrm{NCH}_{2} \mathrm{C}=\mathrm{O}\right), 9.17\left(2 \mathrm{H}, \mathrm{s}, 2 \mathrm{NHNH}_{2}\right) ;{ }^{13} \mathrm{C}-\mathrm{NMR}(\delta \mathrm{ppm}): 14.54$ $\left(2 \mathrm{CH}_{3}\right), 20.37\left(2 \mathrm{NCH}_{2} \mathrm{CH}_{2} \mathrm{CH}_{2}\right), 28.47\left(2 \mathrm{NCH}_{2} \underline{\mathrm{C}}_{2}\right), 43.87\left(2 \mathrm{NCH}_{2}\right), 49.35\left(2 \mathrm{NCH}_{2} \mathrm{C}=\mathrm{O}\right), 146.87$ $(2 \mathrm{C}=\mathrm{N}), 154.99(2 \mathrm{C}=\mathrm{O}), 171.45(2 \mathrm{C}=\mathrm{O}$ hydrazide $)$, MS (ESI): $m / z(\%) 396.20\left(\mathrm{M}^{+1}\right)$.

\subsection{General Method for the Synthesis of Compounds 4-6}

A mixture of corresponding compound $3(10 \mathrm{mmol})$ and 4-fluorophenylisothiocyanate (for compounds 4), 4-bromophenylisothiocyanate (for compounds 5) or p-tolylisothiocyanate (for compounds 6) (15 mmol) was refluxed in ethanol for $4 \mathrm{~h}$. The solution was cooled and a white solid appeared. This was filtered and recrystallized from ethanol to afford the desired product.

N-(4-Fluorophenyl)-2-(2-(4-(4-(1-(2-(2-(4-fluorophenylcarbamothioyl)hydrazinyl)-2-oxoethyl)-3methyl-5-oxo-1H-1,2,4-triazole-4(5H)-yl)butyl)-3-methyl-5-oxo-4,5-dihydro-1,2,4-triazole-1yl)acetyl)hydrazinecarbothioamide (4a). Yield 85\%, m.p. $180-181{ }^{\circ} \mathrm{C}$. IR $\left(\mathrm{v}, \mathrm{cm}^{-1}\right): 3,245$ (NH), 
1,698 (triazole $\mathrm{C}=\mathrm{O}$ ), $1610(\mathrm{C}=\mathrm{N}), 1191(\mathrm{C}=\mathrm{S})$; Anal. Calcd (\%) for $\mathrm{C}_{28} \mathrm{H}_{32} \mathrm{~F}_{2} \mathrm{~N}_{12} \mathrm{O}_{4} \mathrm{~S}_{2}$ : C, 47.85; $\mathrm{H}$, 4.59; N, 23.92. Found: C, 47.89; H, 4.65; N, 23.97; ${ }^{1} \mathrm{H}-\mathrm{NMR}(\delta \mathrm{ppm}): 1.58\left(4 \mathrm{H}, \mathrm{bs}, 2 \mathrm{NCH}_{2} \underline{\mathrm{C}}_{2}\right)$, $2.47\left(6 \mathrm{H}, \mathrm{s}, 2 \mathrm{CH}_{3}\right), 3.56\left(4 \mathrm{H}, \mathrm{t}, 2 \mathrm{NCH}_{2}\right), 4.41\left(4 \mathrm{H}, \mathrm{s}, 2 \mathrm{NCH}_{2} \mathrm{C}=\mathrm{O}\right), 7.11-7.20(4 \mathrm{H}, \mathrm{m}, \mathrm{ArH})$, 7.33-7.40 (4H, m, ArH), $9.63(2 \mathrm{H}, \mathrm{s}, \mathrm{NH}), 9.74(2 \mathrm{H}, \mathrm{s}, \mathrm{NH}), 10.26(2 \mathrm{H}, \mathrm{s}, \mathrm{NH}) ;{ }^{13} \mathrm{C}-\mathrm{NMR}(\delta \mathrm{ppm})$ : $11.94\left(2 \mathrm{CH}_{3}\right), 26.17\left(2 \mathrm{NCH}_{2} \underline{\mathrm{CH}_{2}}\right), 46.89\left(2 \mathrm{NCH}_{2} \mathrm{CH}_{2}\right), 56.71\left(2 \mathrm{NCH}_{2} \mathrm{C}=\mathrm{O}\right), \operatorname{ArC}:[115.28(2 \mathrm{CH})$, $128.78(2 \mathrm{CH}), 135.95(2 \mathrm{C}), 144.47(2 \mathrm{C})], 154.66(2 \mathrm{C}=\mathrm{N}), 162.66(2$ triazole $\mathrm{C}=\mathrm{O}), 167.32(2 \mathrm{C}=\mathrm{O})$, $181.61(2 \mathrm{C}=\mathrm{S})$; MS (ESI): $m / z(\%) 703.20(\mathrm{M}+1)$.

N-(4-Fluorophenyl)-2-(2-(4-(6-(1-(2-(2-(4-fluorophenylcarbonothioyl)hydrazinyl)-2-oxoethyl)-3methyl-5-oxo-4,5-dihydro-1,2,4-triazole-4(5H)-yl)hexyl)-3-methyl-5-oxo-4,5-dihydro-1,2,4-triazole-1yl)acetyl)hydrazinecarbothioamide (4b). Yield 80\%, m.p. 170-171 ${ }^{\circ} \mathrm{C}$. IR (v, $\left.\mathrm{cm}^{-1}\right): 3234(\mathrm{NH}), 1688$ (triazole $\mathrm{C}=\mathrm{O}$ ), $1610(\mathrm{C}=\mathrm{N}), 1189(\mathrm{C}=\mathrm{S})$; Anal. Calcd $(\%)$ for $\mathrm{C}_{30} \mathrm{H}_{36} \mathrm{~F}_{2} \mathrm{~N}_{12} \mathrm{O}_{4} \mathrm{~S}_{2}: \mathrm{C}, 49.30 ; \mathrm{H}, 4.97 ; \mathrm{N}$, 23.00. Found: C, 49.36; H, 4.92; N, 23.07; ${ }^{1} \mathrm{H}-\mathrm{NMR}(\delta \mathrm{ppm}): 1.15\left(4 \mathrm{H}, \mathrm{bs}, 2 \mathrm{NCH}_{2} \mathrm{CH}_{2} \mathrm{CH}_{2}\right), 1.51$ $\left(4 \mathrm{H}, \mathrm{bs}, 2 \mathrm{NCH}_{2} \mathrm{CH}_{2}\right), 2.14\left(6 \mathrm{H}, \mathrm{s}, 2 \mathrm{CH}_{3}\right), 3.52\left(4 \mathrm{H}, \mathrm{bs}, 2 \mathrm{NCH}_{2}\right), 4.02\left(4 \mathrm{H}, \mathrm{bs}, 2 \mathrm{NCH}_{2} \mathrm{C}=\mathrm{O}\right)$, 6.76-7.45 (8H, m, ArH), $9.58(2 \mathrm{H}, \mathrm{s}, \mathrm{NH}), 9.81(2 \mathrm{H}, \mathrm{s}, \mathrm{NH}), 10.74(2 \mathrm{H}, \mathrm{s}, \mathrm{NH}) ;{ }^{13} \mathrm{C}-\mathrm{NMR}(\delta \mathrm{ppm})$ : $11.86\left(\mathrm{CH}_{3}\right), 20.45\left(2 \mathrm{NCH}_{2} \mathrm{CH}_{2} \mathrm{CH}_{2}\right), 27.19\left(\mathrm{NCH}_{2} \underline{\mathrm{CH}}_{2}\right), 45.93\left(\mathrm{NCH}_{2} \mathrm{CH}_{2}\right), 55.78\left(\mathrm{NCH}_{2} \mathrm{C}=\mathrm{O}\right), \operatorname{ArC}$ : [115.72 (2CH), $128.95(2 \mathrm{CH}), 136.05(2 \mathrm{C}), 144.90(2 \mathrm{C})], 154.86(2 \mathrm{C}=\mathrm{N}), 162.68(2$ triazole $\mathrm{C}=\mathrm{O})$, $168.60(2 \mathrm{C}=\mathrm{O}), 181.64(2 \mathrm{C}=\mathrm{S})$; MS (ESI): $m / z(\%) 730.24(\mathrm{M}+1)$.

N-(4-Bromophenyl)-2-(2-(4-(4-(1-(2-(2-(4-bromophenylcarbamothioyl)hydrazinyl)-2-oxoethyl)-3methyl-5-oxo-1H-1,2,4-triazole-4(5H)-yl)butyl)-3-methyl-5-oxo-4,5-dihydro-1,2,4-triazole-1-yl)acetyl)hydrazinecarbothioamide (5a). Yield 76\%, m.p. 206-207 ${ }^{\circ} \mathrm{C}$. IR (v, $\left.\mathrm{cm}^{-1}\right): 3265(\mathrm{NH}), 1705$ (triazole $\mathrm{C}=\mathrm{O}$ ), $1587(\mathrm{C}=\mathrm{N}), 1192(\mathrm{C}=\mathrm{S})$; Anal. Calcd (\%) for $\mathrm{C}_{28} \mathrm{H}_{32} \mathrm{Br}_{2} \mathrm{~N}_{12} \mathrm{O}_{4} \mathrm{~S}_{2}$ : C, 40.78; H, 3.91; $\mathrm{N}, 20.38$. Found: C, 40.75; H, 3.98; N, 20.42; ${ }^{1} \mathrm{H}-\mathrm{NMR}(\delta \mathrm{ppm}): 1.04\left(4 \mathrm{H}, \mathrm{bs}, 2 \mathrm{NCH}_{2} \mathrm{CH}_{2}\right), 2.16(6 \mathrm{H}$, s, $\left.2 \mathrm{CH}_{3}\right), 3.57\left(4 \mathrm{H}, \mathrm{t}, 2 \mathrm{NCH}_{2}\right), 4.43\left(4 \mathrm{H}, \mathrm{s}, 2 \mathrm{NCH}_{2} \mathrm{C}=\mathrm{O}\right), 7.38-7.55(8 \mathrm{H}, \mathrm{m}, \mathrm{ArH}), 9.67(2 \mathrm{H}, \mathrm{s}$, $2 \mathrm{NH}), 9.83(2 \mathrm{H}, \mathrm{s}, 2 \mathrm{NH}), 10.30(2 \mathrm{H}, \mathrm{s}, 2 \mathrm{NH}) ;{ }^{13} \mathrm{C}-\mathrm{NMR}(\delta \mathrm{ppm}): 11.25\left(\mathrm{CH}_{3}\right), 25.45\left(\mathrm{NCH}_{2} \mathrm{CH}_{2}\right)$, 46.37( $\left.\mathrm{NCH}_{2} \mathrm{CH}_{2}\right), 56.79\left(\mathrm{NCH}_{2} \mathrm{C}=\mathrm{O}\right), \operatorname{ArC}$ : [115.34 (2CH), $\left.128.86(2 \mathrm{CH}), 135.98(2 \mathrm{C}), 144.46(2 \mathrm{C})\right]$, $154.67(2 \mathrm{C}=\mathrm{N}), 162.69(2$ triazole $\mathrm{C}=\mathrm{O}), 167.35(2 \mathrm{C}=\mathrm{O}), 181.62(2 \mathrm{C}=\mathrm{S}) ; \mathrm{MS}(\mathrm{ESI}): m / z(\%)$ $824.05\left(\mathrm{M}^{+}\right)$.

N-(4-Bromophenyl)-2-(2-(4-(6-(1-(2-(2-(4-bromophenylcarbonothioyl)hydrazinyl)-2-oxoethyl)-3methyl-5-oxo-4,5-dihydro-1,2,4-triazole-4(5H)-yl)hexyl)-3-methyl-5-oxo-4,5-dihydro-1,2,4-triazole-1yl)acetyl)hydrazinecarbothioamide (5b). Yield 79\%, m.p. 190-191 ${ }^{\circ} \mathrm{C}$. IR (v, $\left.\mathrm{cm}^{-1}\right): 3224(\mathrm{NH}), 1711$ (triazole $\mathrm{C}=\mathrm{O}$ ), 1668 (hydrazide $\mathrm{C}=\mathrm{O}$ ), $1185(\mathrm{C}=\mathrm{S})$; Anal. Calcd (\%) for $\mathrm{C}_{30} \mathrm{H}_{36} \mathrm{Br}_{2} \mathrm{~N}_{12} \mathrm{O}_{4} \mathrm{~S}_{2}$ : C, 42.26; $\mathrm{H}, 4.26$; N, 19.71. Found: $\mathrm{C}, 42.21 ; \mathrm{H}, 4.30 ; \mathrm{N}, 19.76 ;{ }^{1} \mathrm{H}-\mathrm{NMR}(\delta \mathrm{ppm}): 1.27$ (4H, bs, 2 $\left.\mathrm{NCH}_{2} \mathrm{CH}_{2} \mathrm{CH}_{2}\right), 1.56\left(4 \mathrm{H}, \mathrm{bs}, 2 \mathrm{NCH}_{2} \mathrm{CH}_{2}\right), 2.54\left(6 \mathrm{H}, \mathrm{s}, 2 \mathrm{CH}_{3}\right), 3.60\left(4 \mathrm{H}, \mathrm{t}, 2 \mathrm{NCH}_{2}\right), 4.44(4 \mathrm{H}, \mathrm{s}, 2$ $\left.\mathrm{NCH}_{2} \mathrm{C}=\mathrm{O}\right), 7.10-7.25(4 \mathrm{H}, \mathrm{m}, \mathrm{ArH}), 7.38-7.49(4 \mathrm{H}, \mathrm{m}, \mathrm{ArH}), 9.66(2 \mathrm{H}, \mathrm{s}, 2 \mathrm{NH}), 9.76(2 \mathrm{H}, \mathrm{s}, 2 \mathrm{NH})$, $10.29(2 \mathrm{H}, \mathrm{s}, 2 \mathrm{NH}) ;{ }^{13} \mathrm{C}-\mathrm{NMR}(\delta \mathrm{ppm}): 11.94\left(2 \mathrm{CH}_{3}\right), 20.50\left(2 \mathrm{NCH}_{2} \mathrm{CH}_{2} \mathrm{CH}_{2}\right), 26.16\left(2 \mathrm{NCH}_{2} \mathrm{CH}_{2}\right)$, $46.80\left(2 \mathrm{NCH}_{2} \mathrm{CH}_{2}\right), 56.72\left(2 \mathrm{NCH}_{2} \mathrm{C}=\mathrm{O}\right), \operatorname{ArC}:[115.27(2 \mathrm{CH}), 128.74(2 \mathrm{CH}), 135.90(2 \mathrm{C}), 144.41$ $(2 \mathrm{C})], 154.61(2 \mathrm{C}=\mathrm{N}), 162.68(2$ triazole $\mathrm{C}=\mathrm{O}), 168.35(2 \mathrm{C}=\mathrm{O}), 181.64(2 \mathrm{C}=\mathrm{S}) ; \mathrm{MS}(\mathrm{ESI}): m / z(\%)$ $852.08\left(\mathrm{M}^{+}\right)$. 
N-(p-Tolyl))-2-(2-(3-methyl-4-(4-(3-methyl-5-oxo-1-(2-oxo-2-(2-(p-tolylcarbamothioyl)hydrazinyl)ethyl)-1H-1,2,4-triazole-4(5H)-yl)butyl)-5-oxo-4,5-dihydro-1,2,4-triazole-1-yl)acetyl)hydrazinecarbothioamide (6a). Yield 77\%, m.p. 218-219 ${ }^{\circ} \mathrm{C}$. IR (v, $\mathrm{cm}^{-1}$ ): $3224(\mathrm{NH}), 1705$ (triazole C=O), 1637 (hydrazide $\mathrm{C}=\mathrm{O}), 1188(\mathrm{C}=\mathrm{S})$; Anal. Calcd (\%) for $\mathrm{C}_{30} \mathrm{H}_{38} \mathrm{~N}_{12} \mathrm{O}_{4} \mathrm{~S}_{2}$ : C, 51.86; H, 5.51; N, 24.19. Found: C, 51.90; H, 5.57; N, 24.11; ${ }^{1} \mathrm{H}-\mathrm{NMR}(\delta \mathrm{ppm}): 1.58\left(4 \mathrm{H}, \mathrm{bs}, 2 \mathrm{NCH}_{2} \underline{\mathrm{C}}_{2}\right), 2.15\left(6 \mathrm{H}, \mathrm{s}, 2 \mathrm{CH}_{3}\right)$, 2.27 (6H, s, 2 phenyl- $\left.\mathrm{CH}_{3}\right), 3.39\left(4 \mathrm{H}, \mathrm{t}, 2 \mathrm{NCH}_{2}\right), 4.42\left(4 \mathrm{H}, \mathrm{s}, 2 \mathrm{NCH}_{2} \mathrm{C}=\mathrm{O}\right), 7.14-7.23(8 \mathrm{H}, \mathrm{m}, \mathrm{ArH})$, $9.56(2 \mathrm{H}, \mathrm{s}, 2 \mathrm{NH}), 9.65(2 \mathrm{H}, \mathrm{s}, 2 \mathrm{NH}), 10.24(2 \mathrm{H}, \mathrm{s}, 2 \mathrm{NH}) ;{ }^{13} \mathrm{C}-\mathrm{NMR}(\delta \mathrm{ppm}): 11.94\left(\mathrm{CH}_{3}\right), 21.09(2$ phenyl- $\left.\mathrm{CH}_{3}\right), 26.17\left(\mathrm{NCH}_{2} \underline{\mathrm{CH}}_{2}\right), 46.93\left(\mathrm{NCH}_{2} \mathrm{CH}_{2}\right), 58.62\left(\mathrm{NCH}_{2} \mathrm{C}=\mathrm{O}\right), \operatorname{ArC}$ : [120.54 $(2 \mathrm{CH}), 129.27$ $(2 \mathrm{CH}), 137.07(2 \mathrm{C}), 144.47(2 \mathrm{C})], 154.64(2 \mathrm{C}=\mathrm{N}), 167.31(2$ triazole $\mathrm{C}=\mathrm{O}), 167.35(2 \mathrm{C}=\mathrm{O}), 181.34$ $(2 \mathrm{C}=\mathrm{S}) ; \mathrm{MS}(\mathrm{ESI}): \mathrm{m} / z(\%) 694.26\left(\mathrm{M}^{+}\right)$.

N-(p-Tolyl))-2-(2-(3-methyl-4-(4-(3-methyl-5-oxo-1-(2-oxo-2-(2-(p-tolylcarbamothioyl)hydrazinyl)ethyl)-1H-1,2,4-triazole-4(5H)-yl)hexyl)-5-oxo-4,5-dihydro-1,2,4-triazole-1-yl)acetyl)hydrazinecarbothioamide (6b). Yield 76\%, m.p. 185-186 ${ }^{\circ} \mathrm{C}$. IR (v, $\left.\mathrm{cm}^{-1}\right): 3247(\mathrm{NH}), 1688$ (triazole C=O), $1590(\mathrm{C}=\mathrm{N}), 1190(\mathrm{C}=\mathrm{S})$; Anal. Calcd (\%) for $\mathrm{C}_{32} \mathrm{H}_{42} \mathrm{~N}_{12} \mathrm{O}_{4} \mathrm{~S}_{2}: \mathrm{C}, 53.17 ; \mathrm{H}, 5.86 ; \mathrm{N}, 23.25$. Found: $\mathrm{C}$, 53.22 H, 5.92; N, 23.48; ${ }^{1} \mathrm{H}-\mathrm{NMR}(\delta \mathrm{ppm}): 1.26\left(4 \mathrm{H}, \mathrm{bs}, 2 \mathrm{NCH}_{2} \mathrm{CH}_{2} \mathrm{C}_{2}\right), 1.54\left(4 \mathrm{H}, \mathrm{bs}, 2 \mathrm{NCH}_{2} \underline{\mathrm{C}}_{2}\right)$, $2.17\left(6 \mathrm{H}, \mathrm{s}, 2 \mathrm{CH}_{3}\right), 2.29\left(6 \mathrm{H}, \mathrm{s}, 2\right.$ phenyl- $\left.\mathrm{CH}_{3}\right), 3.51\left(4 \mathrm{H}, \mathrm{t}, 2 \mathrm{NCH}_{2}\right), 4.35\left(4 \mathrm{H}, \mathrm{s}, 2 \mathrm{NCH}_{2} \mathrm{C}=\mathrm{O}\right)$, 7.07-7.13 (4H, m, ArH), 7.41-7.64 (4H, m, ArH), $9.61(2 \mathrm{H}, \mathrm{s}, 2 \mathrm{NH}), 9.80(2 \mathrm{H}, \mathrm{s}, 2 \mathrm{NH}), 10.35$ (2H, s, $2 \mathrm{NH}) ;{ }^{13} \mathrm{C}-\mathrm{NMR}(\delta \mathrm{ppm}): 12.86\left(\mathrm{CH}_{3}\right), 20.95\left(2 \mathrm{NCH}_{2} \mathrm{CH}_{2} \mathrm{CH}_{2}\right), 21.00$ (2 phenyl- $\left.\mathrm{CH}_{3}\right), 25.58$ $\left(\mathrm{NCH}_{2} \underline{\mathrm{CH}}_{2}\right), 43.25\left(\mathrm{NCH}_{2} \mathrm{CH}_{2}\right), 56.12\left(\mathrm{NCH}_{2} \mathrm{C}=\mathrm{O}\right), \operatorname{ArC}$ : [120.67 (2CH), $127.65(2 \mathrm{CH}), 138.76(2 \mathrm{C})$, $145.35(2 \mathrm{C})], 153.50(2 \mathrm{C}=\mathrm{N}), 160.21(2$ triazole $\mathrm{C}=\mathrm{O}), 169.67(2 \mathrm{C}=\mathrm{O}), 180.81(2 \mathrm{C}=\mathrm{S}) ; \mathrm{MS}(\mathrm{ESI}): \mathrm{m} / z$ (\%) $722.29\left(\mathrm{M}^{+}\right)$.

\subsection{General Method for the Synthesis of Compounds 7-9}

A mixture of corresponding thiosemicarbazides 4-6 $(10 \mathrm{mmol})$ in cold concentrated sulfuric acid $(30 \mathrm{~mL})$ was stirred for $10 \mathrm{~min}$ then, the mixture was allowed to reach room temperature. After stirring for an additional $30 \mathrm{~min}$, the resulting solution was poured into ice cold water and made alkaline to $\mathrm{pH}$ 8 with ammonia. The precipitated product was filtered, washed with water and recrystallized from ethanol to afford the pure compounds.

4,4'-(Butane-1,4-diyl)bis(2-((5-(4-fluorophenylamino) 1,3,4-thiadiazole-2-yl)methyl)-5-methyl-2H1,2,4-triazole-3(4H)-one) (7a). Yield 88\%, m.p. 300-301 ${ }^{\circ} \mathrm{C}$. IR (v, $\mathrm{cm}^{-1}$ ): $3267(\mathrm{NH}), 1694$ (triazole $\mathrm{C}=\mathrm{O}), 1621(\mathrm{C}=\mathrm{N})$; Anal. Calcd $(\%)$ for $\mathrm{C}_{28} \mathrm{H}_{28} \mathrm{~F}_{2} \mathrm{~N}_{12} \mathrm{O}_{2} \mathrm{~S}_{2}$ : C, 50.44; H, 4.23; N, 25.21. Found: C, 50.49; H, 4.18; N, 25.28; ${ }^{1} \mathrm{H}-\mathrm{NMR}(\delta \mathrm{ppm}): 1.54\left(4 \mathrm{H}, \mathrm{bs}, \mathrm{NCH}_{2} \mathrm{CH}_{2}\right), 2.16\left(6 \mathrm{H}, \mathrm{s}, 2 \mathrm{CH}_{3}\right), 3.58(4 \mathrm{H}, \mathrm{bs}$,

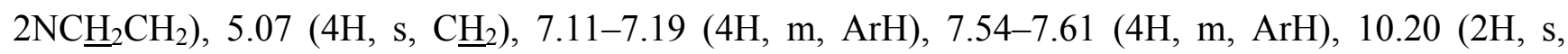
$2 \mathrm{NH}) ;{ }^{13} \mathrm{C}-\mathrm{NMR}(\delta \mathrm{ppm}): 11.98\left(2 \mathrm{CH}_{3}\right), 26.10\left(2 \mathrm{NCH}_{2} \mathrm{CH}_{2}\right), 38.84-41.34\left(\mathrm{DMSO}-d_{6}+2 \mathrm{NCH}_{2} \mathrm{CH}_{2}\right)$, $44.03\left(2 \underline{\mathrm{CH}}_{2}\right), \operatorname{ArC}:[105.00(2 \mathrm{C}), 116.58(2 \mathrm{CH}), 119.89(2 \mathrm{CH}), 137.61(2 \mathrm{C}), 145.25(2 \mathrm{C}), 153.77$ $(2 \mathrm{C})$ ], $155.57(2 \mathrm{C}=\mathrm{N}), 160.32(2$ triazole $\mathrm{C}=\mathrm{O}), \mathrm{MS}(\mathrm{ESI}): \mathrm{m} / z(\%) 666.19\left(\mathrm{M}^{+}\right)$.

4,4'-(Hexane-1,6-diyl)bis(2-((5-(4-fluorophenylamino)1,3,4-thiadiazole-2-yl)methyl)-5-methyl-2H1,2,4-triazole-3(4H)-one) (7b). Yield 85\%, m.p. 278-279 ${ }^{\circ} \mathrm{C}$. IR (v, $\mathrm{cm}^{-1}$ ): $3219(\mathrm{NH}), 1691$ (triazole $\mathrm{C}=\mathrm{O}), 1,627(\mathrm{C}=\mathrm{N})$; Anal. Calcd (\%) for $\mathrm{C}_{30} \mathrm{H}_{32} \mathrm{~F}_{2} \mathrm{~N}_{12} \mathrm{O}_{2} \mathrm{~S}_{2}$ : C, 51.86; H, 4.64; N, 24.19. Found: C, 
51.82; H, 4.68; N, 24.12; ${ }^{1} \mathrm{H}-\mathrm{NMR}(\delta \mathrm{ppm}): 1.20\left(4 \mathrm{H}, \mathrm{bs}, 2 \mathrm{NCH}_{2} \mathrm{CH}_{2} \underline{\mathrm{C}}_{2}\right), 1.58\left(4 \mathrm{H}, \mathrm{bs}, \mathrm{NCH}_{2} \mathrm{C}_{2}\right)$, $2.21\left(6 \mathrm{H}, \mathrm{s}, 2 \mathrm{CH}_{3}\right), 3.48\left(4 \mathrm{H}, \mathrm{bs}, 2 \mathrm{NC}_{2} \mathrm{CH}_{2}\right), 5.11\left(4 \mathrm{H}, \mathrm{s}, 2 \underline{\mathrm{C}}_{2}\right), 7.12-7.20(8 \mathrm{H}, \mathrm{m}, \mathrm{ArH}), 10.26$ $(2 \mathrm{H}, \mathrm{s}, 2 \mathrm{NH}) ;{ }^{13} \mathrm{C}-\mathrm{NMR}(\delta \mathrm{ppm}): 11.25\left(2 \mathrm{CH}_{3}\right), 20.98\left(2 \mathrm{NCH}_{2} \mathrm{CH}_{2} \mathrm{CH}_{2}\right), 26.32\left(2 \mathrm{NCH}_{2} \mathrm{CH}_{2}\right)$, 38.80-41.41 (DMSO- $\left.d_{6}+2 \mathrm{NCH}_{2} \mathrm{CH}_{2}\right), 44.56\left(2 \underline{\mathrm{CH}}_{2}\right), \operatorname{ArC}:[101.07(2 \mathrm{C}), 117.45(2 \mathrm{CH}), 120.24$ $(2 \mathrm{CH}), 137.65(2 \mathrm{C}), 145.20(2 \mathrm{C}), 153.76(2 \mathrm{C})], 155.51(2 \mathrm{C}=\mathrm{N}), 160.30$ (2 triazole $\mathrm{C}=\mathrm{O})$, MS (ESI): $m / z(\%) 694.22\left(\mathrm{M}^{+}\right)$.

4,4'-(Butane-1,4-diyl)bis(2-((5-(4-bromophenylamino) 1,3,4-thiadiazole-2-yl)methyl)-5-methyl-2H1,2,4-triazole-3(4H)-one) (8a). Yield 83\%, m.p. 299-300 ${ }^{\circ} \mathrm{C}$. IR (v, $\mathrm{cm}^{-1}$ ): 3241 (NH), 1690 (triazole $\mathrm{C}=\mathrm{O}), 1,603(\mathrm{C}=\mathrm{N})$; Anal. Calcd (\%) for $\mathrm{C}_{28} \mathrm{H}_{28} \mathrm{Br}_{2} \mathrm{~N}_{12} \mathrm{O}_{2} \mathrm{~S}_{2}$ : C, 43.40; H, 3.77; N, 20.94. Found: C, 43.46; H, 3.79; N, 20.98; ${ }^{1} \mathrm{H}-\mathrm{NMR}(\delta \mathrm{ppm}): 1.54\left(4 \mathrm{H}, \mathrm{bs}, \mathrm{NCH}_{2} \mathrm{CH}_{2}\right), 2.16\left(6 \mathrm{H}, \mathrm{s}, 2 \mathrm{CH}_{3}\right), 3.58(4 \mathrm{H}, \mathrm{bs}$, $\left.2 \mathrm{NC}_{2} \mathrm{CH}_{2}\right), 5.07\left(4 \mathrm{H}, \mathrm{s}, \underline{\mathrm{CH}}_{2}\right), 7.11-7.19(8 \mathrm{H}, \mathrm{m}, \mathrm{ArH}), 10.23(2 \mathrm{H}, \mathrm{s}, 2 \mathrm{NH}) ;{ }^{13} \mathrm{C}-\mathrm{NMR}(\delta \mathrm{ppm})$ : $11.19\left(2 \mathrm{CH}_{3}\right), 25.31\left(2 \mathrm{NCH}_{2} \underline{\mathrm{CH}}_{2}\right), 38.84-41.31\left(\mathrm{DMSO}-d_{6}+2 \mathrm{NCH}_{2} \mathrm{CH}_{2}\right), 43.22\left(2 \mathrm{CH}_{2}\right), \operatorname{ArC}:[113.11$ (2C), $119.21(2 \mathrm{CH}), 131.69(2 \mathrm{CH}), 139.59(2 \mathrm{C}), 144.43(2 \mathrm{C}), 152.96(2 \mathrm{C})], 155.02(2 \mathrm{C}=\mathrm{N}), 164.85(2$ triazole $\mathrm{C}=\mathrm{O})$, $\mathrm{MS}(\mathrm{ESI}): \mathrm{m} / z(\%)$ 788.02($\left(\mathrm{M}^{+}\right)$.

4,4'-(Hexane-1,6-diyl)bis(2-((5-(4-bromophenylamino) 1,3,4-thiadiazole-2-yl)methyl)-5-methyl-2H1,2,4-triazole-3(4H)-one) (8b). Yield 80\%, m.p. 243-244 ${ }^{\circ} \mathrm{C}$. IR $\left(v, \mathrm{~cm}^{-1}\right): 3285(\mathrm{NH}), 1703$ (triazole $\mathrm{C}=\mathrm{O}), 1667(\mathrm{C}=\mathrm{N})$; Anal. Calcd (\%) for $\mathrm{C}_{30} \mathrm{H}_{32} \mathrm{Br}_{2} \mathrm{~N}_{12} \mathrm{O}_{2} \mathrm{~S}_{2}: \mathrm{C}, 44.12 ; \mathrm{H}, 3.95 ; \mathrm{N}, 20.58$. Found: C, 44.19; H, 4.05; N, 20.62; ${ }^{1} \mathrm{H}-\mathrm{NMR}$ ( $\left.\delta \mathrm{ppm}\right): 1.24$ (4H, bs, $\left.2 \mathrm{NCH}_{2} \mathrm{CH}_{2} \mathrm{C}_{2}\right), 1.50\left(4 \mathrm{H}, \mathrm{bs}, \mathrm{NCH}_{2} \underline{\mathrm{C}}_{2}\right)$, $2.22\left(6 \mathrm{H}, \mathrm{s}, 2 \mathrm{CH}_{3}\right), 3.45\left(4 \mathrm{H}, \mathrm{bs}, 2 \mathrm{NCH}_{2} \mathrm{CH}_{2}\right), 5.10\left(4 \mathrm{H}, \mathrm{s}, 2 \mathrm{CH}_{2}\right), 7.11-7.20(8 \mathrm{H}, \mathrm{m}, \mathrm{ArH}), 10.28$ $(2 \mathrm{H}, \mathrm{s}, 2 \mathrm{NH}) ;{ }^{13} \mathrm{C}-\mathrm{NMR}(\delta \mathrm{ppm}): 11.02\left(2 \mathrm{CH}_{3}\right), 20.96\left(2 \mathrm{NCH}_{2} \mathrm{CH}_{2} \mathrm{CH}_{2}\right), 24.98\left(2 \mathrm{NCH}_{2} \mathrm{CH}_{2}\right)$, 38.84-41.36 (DMSO- $\left.d_{6}+2 \mathrm{NCH}_{2} \mathrm{CH}_{2}\right), 43.19\left(2 \underline{\mathrm{CH}}_{2}\right), \operatorname{ArC}:[115.15(2 \mathrm{C}), 120.11(2 \mathrm{CH}), 131.60$ $(2 \mathrm{CH}), 139.50(2 \mathrm{C}), 144.35(2 \mathrm{C}), 150.55(2 \mathrm{C})], 154.80(2 \mathrm{C}=\mathrm{N}), 164.80(2$ triazole $\mathrm{C}=\mathrm{O}) \mathrm{MS}(\mathrm{ESI})$ : $\mathrm{m} / \mathrm{z}(\%) 817.07(\mathrm{M}+1)$.

4,4'-(Butane-1,4-diyl)bis(2-((5-(p-toluidino)1,3,4-thiadiazole-2-yl)methyl)-5-methyl-2H-1,2,4-triazole3(4H)-one) (9a). Yield 76\%, m.p. 303-304 ${ }^{\circ} \mathrm{C}$. IR (v, $\left.\mathrm{cm}^{-1}\right): 3240(\mathrm{NH}), 1692$ (triazole C=O), 1645 $(\mathrm{C}=\mathrm{N})$; Anal. Calcd (\%) for $\mathrm{C}_{30} \mathrm{H}_{34} \mathrm{~N}_{12} \mathrm{O}_{2} \mathrm{~S}_{2}$ : C, 54.69; H, 5.20; N, 25.51. Found: C, 54.60; H, 5.27; N, 25.58; ${ }^{1} \mathrm{H}-\mathrm{NMR}(\delta \mathrm{ppm}): 1.54\left(4 \mathrm{H}, \mathrm{bs}, \mathrm{NCH}_{2} \mathrm{CH}_{2}\right), 2.18\left(6 \mathrm{H}, \mathrm{s}, 2 \mathrm{CH}_{3}\right), 2.23\left(6 \mathrm{H}, \mathrm{s}, 2\right.$ phenyl- $\left.\mathrm{CH}_{3}\right)$, $3.59\left(4 \mathrm{H}, \mathrm{bs}, 2 \mathrm{NCH}_{2} \mathrm{CH}_{2}\right), 5.07\left(4 \mathrm{H}, \mathrm{s}, 2 \mathrm{CH}_{2}\right), 7.13-7.45(8 \mathrm{H}, \mathrm{m}, \mathrm{ArH}), 10.24(2 \mathrm{H}, \mathrm{s}, 2 \mathrm{NH})$; ${ }^{13} \mathrm{C}-\mathrm{NMR}(\delta \mathrm{ppm}): 12.00\left(2 \mathrm{CH}_{3}\right), 21.03\left(2\right.$ phenyl- $\left.\mathrm{CH}_{3}\right), 26.14\left(2 \mathrm{NCH}_{2} \mathrm{CH}_{2}\right), 38.84-41.35\left(\mathrm{DMSO}-d_{6}\right.$ $\left.+2 \mathrm{NCH}_{2} \mathrm{CH}_{2}\right), 44.07\left(2 \mathrm{CH}_{2}\right), \operatorname{ArC}:[106.27(2 \mathrm{C}), 129.74(2 \mathrm{CH}), 130.20(\mathrm{CH}), 138.83(2 \mathrm{C}), 145.20$ (2C), $153.54(2 \mathrm{C})], 154.96(2 \mathrm{C}=\mathrm{N}), 166.33$ (2 triazole $\mathrm{C}=\mathrm{O}), \mathrm{MS}(\mathrm{ESI}): \mathrm{m} / z(\%) 658.24\left(\mathrm{M}^{+}\right)$.

4,4'-(Hexane-1,6-diyl)bis(2-((5-(p-toluidino)1,3,4-thiadiazole-2-yl)methyl)-5-methyl-2H-1,2,4-triazole3(4H)-one) (9b). Yield 77\%, m.p. 269-270 ${ }^{\circ} \mathrm{C}$. IR (v, $\left.\mathrm{cm}^{-1}\right): 3198(\mathrm{NH}), 1690$ (triazole C=O), 1656 $(\mathrm{C}=\mathrm{N})$; Anal. Calcd (\%) for $\mathrm{C}_{32} \mathrm{H}_{38} \mathrm{~N}_{12} \mathrm{O}_{2} \mathrm{~S}_{2}$ : C, 55.96; H, 5.58; N, 24.47. Found: C, 56.04; H, 5.68; N, $24.40{ }^{1} \mathrm{H}-\mathrm{NMR}(\delta \mathrm{ppm}): 1.24\left(4 \mathrm{H}, \mathrm{bs}, 2 \mathrm{NCH}_{2} \mathrm{CH}_{2} \mathrm{CH}_{2}\right), 2.25\left(6 \mathrm{H}, \mathrm{s}, 2\right.$ phenyl- $\left.\mathrm{CH}_{3}\right), 1.50$ (4H, bs,

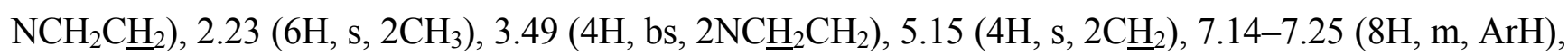
$10.27(2 \mathrm{H}, \mathrm{s}, 2 \mathrm{NH}) ;{ }^{13} \mathrm{C}-\mathrm{NMR}(\delta \mathrm{ppm}): 12.01\left(2 \mathrm{CH}_{3}\right), 20.94\left(2 \mathrm{NCH}_{2} \mathrm{CH}_{2} \mathrm{CH}_{2}\right), 21.03\left(2\right.$ phenyl- $\left.\mathrm{CH}_{3}\right)$, $26.18\left(2 \mathrm{NCH}_{2} \underline{\mathrm{CH}}_{2}\right), 38.80-41.39\left(\mathrm{DMSO}-d_{6}+2 \mathrm{NCH}_{2} \mathrm{CH}_{2}\right), 44.07\left(2 \underline{\mathrm{CH}}_{2}\right), \operatorname{ArC}:[108.27(2 \mathrm{C}), 128.79$ 
$(2 \mathrm{CH}), 130.21(\mathrm{CH}), 138.73(2 \mathrm{C}), 145.28(2 \mathrm{C}), 150.05(2 \mathrm{C})], 154.00(2 \mathrm{C}=\mathrm{N}), 167.56(2$ triazole $\mathrm{C}=\mathrm{O}), \mathrm{MS}(\mathrm{ESI}): m / z(\%) 687.27(\mathrm{M}+1)$.

\subsection{General Method for the Synthesis of Compounds $\mathbf{1 0}$ and $\mathbf{1 1}$}

A solution of corresponding carbothioamide (4-6) (10 mmol) inequivalent amount of $2 \mathrm{~N} \mathrm{NaOH}$ solution was refluxed for $3 \mathrm{~h}$. The resulting solution was cooled to room temperature and acidified topH 3-4 with $37 \% \mathrm{HCl}$. The precipitate formed was filtered, washedwith water and recrystallized from dimethyl sulfoxide/water (1:1) to afford the desired compound.

4,4'-(Butane-1,4-diyl)bis(2-((4-(4-fluorophenyl)-5-mercapto-4H-1,2,4-triazole-3-yl)methyl)-5-methyl2H-1,2,4-triazole-3(4H)-one) (10a). Yield 89\%, m.p. 304-305 ${ }^{\circ} \mathrm{C}$. IR (v, $\left.\mathrm{cm}^{-1}\right): 2962(\mathrm{SH}), 1676$ (triazole $\mathrm{C}=\mathrm{O}$ ), $1589\left(\mathrm{C}=\mathrm{N}\right.$ ); Anal. Calcd (\%) for $\mathrm{C}_{28} \mathrm{H}_{28} \mathrm{~F}_{2} \mathrm{~N}_{12} \mathrm{O}_{2} \mathrm{~S}_{2}$ : C, 50.44; $\mathrm{H}, 4.23$; N, 25.21 . Found: C, 50.48; H, 4.27; N, 25.28; ${ }^{1} \mathrm{H}-\mathrm{NMR}(\delta \mathrm{ppm}): 1.27\left(4 \mathrm{H}, \mathrm{bs}, \mathrm{NCH}_{2} \mathrm{CH}_{2}\right), 2.05\left(6 \mathrm{H}, \mathrm{s}, 2 \mathrm{CH}_{3}\right)$, $3.46\left(4 \mathrm{H}, \mathrm{bs}, 2 \mathrm{NC}_{2} \mathrm{CH}_{2}\right), 4.79\left(4 \mathrm{H}, \mathrm{s}, 2 \mathrm{CH}_{2}\right), 7.23-7.26(8 \mathrm{H}, \mathrm{m}, 2 \mathrm{ArH}), 13.95(2 \mathrm{H}, \mathrm{s}, 2 \mathrm{SH})$; ${ }^{13} \mathrm{C}-\mathrm{NMR}(\delta \mathrm{ppm}): 11.71\left(2 \mathrm{CH}_{3}\right), 26.08\left(2 \mathrm{NCH}_{2} \underline{\mathrm{CH}}_{2}\right), 38.82-41.31\left(\mathrm{DMSO}-d_{6}+2 \mathrm{NCH}_{2} \mathrm{CH}^{2}+2 \underline{\mathrm{CH}_{2}}\right)$, ArC: [116.62 (2C), $129.84(2 \mathrm{C}), 130.43(\mathrm{CH}) 145.04(2 \mathrm{CH}), 148.54$ (2C), 153.08 (2C)], 165.32 $(2 \mathrm{C}=\mathrm{N}), 169.27(2$ triazole $\mathrm{C}=\mathrm{O}), \mathrm{MS}(\mathrm{ESI}): m / z(\%) 667.19\left(\mathrm{M}^{+}\right)$.

4,4'-(Hexane-1,6-diyl)bis(2-((4-(4-fluorophenyl)-5-mercapto-4H-1,2,4-triazole-3-yl)methyl)-5-methyl2H-1,2,4-triazole-3(4H)-one) (10b). Yield 81\%, m.p. 310-311 ${ }^{\circ} \mathrm{C}$. IR (v, $\left.\mathrm{cm}^{-1}\right): 2988(\mathrm{SH}), 1678$ (triazole $\mathrm{C}=\mathrm{O}$ ), $1580\left(\mathrm{C}=\mathrm{N}\right.$ ); Anal. Calcd (\%) for $\mathrm{C}_{30} \mathrm{H}_{32} \mathrm{~F}_{2} \mathrm{~N}_{12} \mathrm{O}_{2} \mathrm{~S}_{2}$ : C, 51.86; H, 4.64; N, 24.19 . Found: C, 51.82; H, 4.72; N, 24.10; ${ }^{1} \mathrm{H}-\mathrm{NMR}(\delta \mathrm{ppm}): 1.22\left(4 \mathrm{H}, \mathrm{bs}, 2 \mathrm{NCH}_{2} \mathrm{CH}_{2} \mathrm{CH}_{2}\right), 1.49(4 \mathrm{H}, \mathrm{bs}$, $\left.2 \mathrm{NCH}_{2} \mathrm{CH}_{2}\right), 2.59\left(6 \mathrm{H}, \mathrm{s}, 2 \mathrm{CH}_{3}\right), 3.65\left(4 \mathrm{H}, \mathrm{t}, 2 \mathrm{NCH}_{2}\right), 7.46-7.65(8 \mathrm{H}, \mathrm{m}, 2 \mathrm{ArH}), 13.90(2 \mathrm{H}, \mathrm{s}$, $2 \mathrm{SH}) ;{ }^{13} \mathrm{C}-\mathrm{NMR}(\delta \mathrm{ppm}): 11.88\left(2 \mathrm{CH}_{3}\right), 20.56\left(2 \mathrm{NCH}_{2} \mathrm{CH}_{2} \underline{\mathrm{C}}_{2}\right), 27.18\left(2 \mathrm{NCH}_{2} \underline{\mathrm{CH}}_{2}\right), 38.79-42.31$ (DMSO- $\left.d_{\sigma}+2 \mathrm{NCH}_{2} \mathrm{CH}_{2}+2 \underline{\mathrm{CH}}_{2}\right), \operatorname{ArC}$ : [119.25 (2C), $126.67(2 \mathrm{C}), 131.46(\mathrm{CH}) 147.25(2 \mathrm{CH}), 149.74$ (2C), $155.01(2 \mathrm{C})], 164.78(2 \mathrm{C}=\mathrm{N}), 169.27$ (2 triazole $\mathrm{C}=\mathrm{O}), \mathrm{MS}(\mathrm{ESI}): \mathrm{m} / z(\%)$ 694.22( $\left.\mathrm{M}^{+}\right)$.

4,4'-(Butane-1,4-diyl)bis(2-((4-(4-bromophenyl)-5-mercapto-4H-1,2,4-triazole-3-yl)methyl)-5-methyl2H-1,2,4-triazole-3(4H)-one) (11a). Yield 91\%, m.p. 296-297 ${ }^{\circ} \mathrm{C}$. IR (v, $\left.\mathrm{cm}^{-1}\right): 2971$ (SH), 1697 (triazole $\mathrm{C}=\mathrm{O}$ ), 1,591 $(\mathrm{C}=\mathrm{N})$; Anal. Calcd (\%) for $\mathrm{C}_{28} \mathrm{H}_{28} \mathrm{Br}_{2} \mathrm{~N}_{12} \mathrm{O}_{2} \mathrm{~S}_{2}: \mathrm{C}$, 42.65; H, 3.58; N, 21.32. Found: C, 42.70; H, 3.65; N, 21.28; ${ }^{1} \mathrm{H}-\mathrm{NMR}(\delta \mathrm{ppm}): 1.29\left(4 \mathrm{H}, \mathrm{bs}, \mathrm{NCH}_{2} \mathrm{C}_{2}\right), 2.07\left(6 \mathrm{H}, \mathrm{s}, 2 \mathrm{CH}_{3}\right)$, $3.36\left(4 \mathrm{H}, \mathrm{bs}, 2 \mathrm{NC}_{2} \mathrm{CH}_{2}\right), 4.84\left(4 \mathrm{H}, \mathrm{s}, 2 \mathrm{NCH}_{2} \mathrm{C}=\mathrm{O}\right), 7.16-7.66(8 \mathrm{H}, \mathrm{m}, 2 \mathrm{ArH}), 13.99(2 \mathrm{H}, \mathrm{s}, 2 \mathrm{SH})$;

${ }^{13} \mathrm{C}-\mathrm{NMR}(\delta \mathrm{ppm}): 11.04\left(2 \mathrm{CH}_{3}\right), 25.43\left(2 \mathrm{NCH}_{2} \mathrm{CH}_{2}\right), 38.08-40.59\left(\mathrm{DMSO}-d_{6}+2 \mathrm{NCH}_{2} \mathrm{CH}_{2}+2 \mathrm{CH}_{2}\right)$, ArC: [122.79 (2C), $129.56(2 \mathrm{C}), 132.14(\mathrm{CH}) 132.22(2 \mathrm{CH}), 144.36(2 \mathrm{C}), 147.73$ (2C)], 152.29 $(2 \mathrm{C}=\mathrm{N}), 168.36(2$ triazole $\mathrm{C}=\mathrm{O}), \mathrm{MS}(\mathrm{ESI}): m / z(\%)$ 788.02 $\left(\mathrm{M}^{+}\right)$.

4,4'-(Hexane-1,6-diyl)bis(2-((4-(4-bromophenyl)-5-mercapto-4H-1,2,4-triazole-3-yl)methyl)-5-methyl2H-1,2,4-triazole-3(4H)-one) (11b). Yield 89\%, m.p. 314-315 ${ }^{\circ} \mathrm{C}$. IR (v, $\left.\mathrm{cm}^{-1}\right): 2969(\mathrm{SH}), 1680$ (triazole $\mathrm{C}=\mathrm{O}$ ), $1578\left(\mathrm{C}=\mathrm{N}\right.$ ); Anal. Calcd (\%) for $\mathrm{C}_{30} \mathrm{H}_{32} \mathrm{Br}_{2} \mathrm{~N}_{12} \mathrm{O}_{2} \mathrm{~S}_{2}: \mathrm{C}, 44.12 ; \mathrm{H}, 3.95 ; \mathrm{N}, 20.58$. Found: C, 44.19; H, 34.07; N, 21.64; ${ }^{1} \mathrm{H}-\mathrm{NMR}(\delta \mathrm{ppm}): 1.20\left(4 \mathrm{H}, \mathrm{bs}, 2 \mathrm{NCH}_{2} \mathrm{CH}_{2} \mathrm{C}_{2}\right), 1.44(4 \mathrm{H}, \mathrm{bs}$, $\left.2 \mathrm{NCH}_{2} \mathrm{CH}_{2}\right), 2.50\left(6 \mathrm{H}, \mathrm{s}, 2 \mathrm{CH}_{3}\right), 3.71\left(4 \mathrm{H}, \mathrm{t}, 2 \mathrm{NCH}_{2}\right), 7.49-7.69(8 \mathrm{H}, \mathrm{m}, 2 \mathrm{ArH}), 13.88$ (2H, s, 2SH); ${ }^{13} \mathrm{C}-\mathrm{NMR}(\delta \mathrm{ppm}): 11.61\left(2 \mathrm{CH}_{3}\right), 20.45 \quad\left(2 \mathrm{NCH}_{2} \mathrm{CH}_{2} \mathrm{CH}_{2}\right), 27.01 \quad\left(2 \mathrm{NCH}_{2} \underline{\mathrm{CH}}_{2}\right), \quad 38.80-42.37$ 
(DMSO- $\left.d_{\sigma}+2 \mathrm{NCH}_{2} \mathrm{CH}_{2}+2 \underline{\mathrm{CH}}_{2}\right), \operatorname{ArC}:[122.88(2 \mathrm{C}), 129.79(2 \mathrm{C}), 132.16(\mathrm{CH}) 132.69(2 \mathrm{CH}), 144.39$ (2C) $147.60(2 \mathrm{C})], 152.41(2 \mathrm{C}=\mathrm{N}), 167.76(2$ triazole $\mathrm{C}=\mathrm{O}), \mathrm{MS}(\mathrm{ESI}): \mathrm{m} / z(\%)$ 816.06( $\left.\mathrm{M}^{+}\right)$.

\subsection{Antioxidant Activity}

DPPH assay: The hydrogen atoms or electrons donation ability of the samples was measured from the bleaching of purple coloured methanol solution of DPPH. This spectrophotometric assay uses stable radical 2,2'-diphenylpicrylhydrazyl (DPPH) as a reagent [21,22]. Fifty microliters of various concentrations of the samples in methanol was added to a $0.004 \%$ methanol solution of DPPH (5 mL). After a 30 min incubation period at room temperature the absorbance was read against a blank at $517 \mathrm{~nm}$. Inhibition free radical DPPH in percent $(I \%)$ was calculated in following way: $I \%$ : $\left(\mathrm{A}_{\text {blank }}-\right.$ $\left.\mathrm{A}_{\text {sample }} / \mathrm{A}_{\text {blank }}\right) \times 100$ where $\mathrm{A}_{\text {blank }}$ is the absorbance of the control reaction (containing all reagents except the test sample), and $\mathrm{A}_{\text {sample }}$ is the absorbance of the test compound. Sample concentration providing $50 \%$ inhibition $\left(\mathrm{IC}_{50}\right)$ was calculated form the graph plotted inhibition percentage against extract concentration. Tests were carried out in triplicate. Butylated hydroxytoluene (BHT) was used as positive control. The results are shown in the Table 1 and Figure 1.

Table 1. $\mathrm{IC}_{50}$ values of compounds $4 \mathbf{a}, \mathbf{b}, \mathbf{5 a}, \mathbf{b}, \mathbf{6 a}, \mathbf{b}, \mathbf{1 0 a}, \mathbf{b}$ and $\mathbf{1 1 a}, \mathbf{b}$.

\begin{tabular}{|c|c|}
\hline Compounds & $\mathrm{DPPH} \mathrm{IC}_{50}(\mu \mathrm{g} / \mathrm{mL}) \pm 0.5$ \\
\hline $4 a$ & $4.7 \pm 0.7$ \\
\hline $4 b$ & $5.6 \pm 0.4$ \\
\hline $5 \mathbf{a}$ & $7.0 \pm 5.7$ \\
\hline $5 \mathbf{b}$ & $7.1 \pm 0.4$ \\
\hline $6 a$ & $4.1 \pm 0.5$ \\
\hline $6 \mathbf{b}$ & $5.1 \pm 0.3$ \\
\hline $10 \mathrm{a}$ & $40 \pm 2.7$ \\
\hline 10b & $40 \pm 0.9$ \\
\hline $11 \mathrm{a}$ & $36 \pm 0.9$ \\
\hline $11 \mathrm{~b}$ & $10 \pm 0.7$ \\
\hline BHT (Positive control) & $19.8 \pm 0.5$ \\
\hline
\end{tabular}

Figure 1. The graphical representation of antioxidant activities compounds.

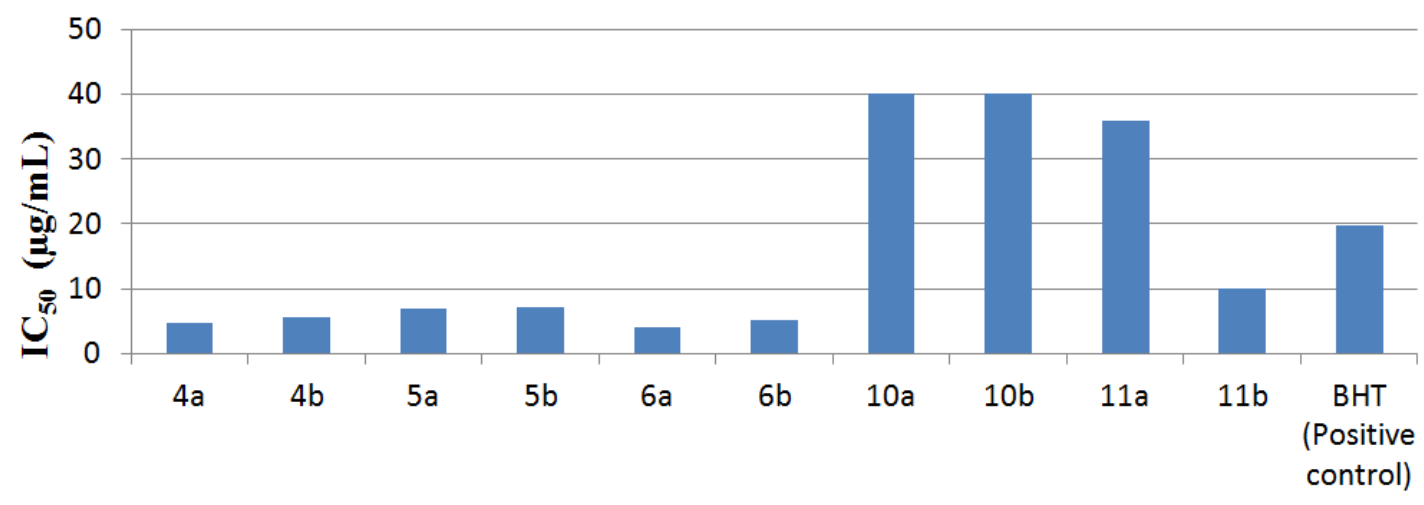




\subsection{Antimicrobial Activity}

All test microorganisms were obtained from the Hifzissiha Institute of Refik Saydam (Ankara, Turkey) and are as follows; Ec; Escherichia coli ATCC 25922, Sa; Staphylococcus aureus ATCC 25923, Pa; Pseudomonas aeruginosa ATCC 27853, Ah; Acinetobacter haemolyticus ATCC 19002, Bs; Bacillus subtilis ATCC 6633, Pv; Proteus vulgaris ATCC 13315, Entc; Enterobacter cloacea ATCC 13047, Ef; Enterococcus faecalis ATCC 29212, Ca; Candida albicans ATCC 60193, Af; Aspergillus sp., Fusa; Fusarium sp., Rhyso; Rhizopus sp. The new compounds were dissolved in dimethylsulphoxide (DMSO) to prepare extract stock solutions of $1,000 \mu \mathrm{g} / \mathrm{mL}$.

Agar Well Diffusion Method

Simple susceptibility screening test using the agar-well diffusion method [23] as adapted earlier was used. Each microorganism was suspended in Brain Heart Infusion (BHI) (Difco, Detroit, MI, USA) broth and diluted to $10^{6}$ colony forming unit (cfu) per mL. They were "flood-inoculated" onto the surface of BHI agar and Sabouraud Dextrose Agar (SDA, Difco) and then dried. For C. albicans, C. tropicalis, Penicillum spp. and Aspergillus spp., SDA was used. Five millimeter diameter wells were cut from the agar using a sterile cork-borer, and $250-5,000 \mu \mathrm{g} / 50 \mu \mathrm{L}$ of the chemical substances were delivered into the wells. The plates were incubated for $18 \mathrm{~h}$ at $35^{\circ} \mathrm{C}$. Antimicrobial activity was evaluated by measuring the zone of inhibition against the test organism. Ceftazidime (Fortum) $(10 \mu \mathrm{g})$ and triflucan $(5 \mu \mathrm{g})$ were standard drugs. The results are shown in the Table 2.

Table 2. Screening for antimicrobial activity of the compounds.

\begin{tabular}{|c|c|c|c|c|c|c|c|c|c|c|c|c|c|}
\hline \multirow{2}{*}{ Compounds } & \multirow{2}{*}{$\begin{array}{l}\text { Stock Concentration } \\
\mu \mathrm{g} / \mathrm{mL}\end{array}$} & \multicolumn{12}{|c|}{ Microorganisms and İnhibition zones (mm) } \\
\hline & & Ec & Sa & $\mathbf{P a}$ & Ah & Bs & Pv & Entc & Ef & $\mathbf{C a}$ & Af & Fusa & Rhyso \\
\hline $2 \mathbf{a}$ & 1000 & - & - & - & 13 & - & - & - & - & 13 & - & - & - \\
\hline 3a & 1000 & - & - & - & 13 & - & - & - & - & - & - & - & - \\
\hline $4 a$ & 1000 & - & - & - & 12 & - & - & - & - & 13 & - & - & - \\
\hline $4 b$ & 1000 & - & - & - & - & - & - & - & - & 11 & - & - & - \\
\hline $5 a$ & 1000 & - & - & 9 & 12 & - & - & - & - & 11 & - & - & - \\
\hline $5 b$ & 1000 & - & - & - & - & - & - & 12 & - & - & - & - & - \\
\hline 6a & 1000 & - & - & - & 12 & - & 11 & - & - & 11 & - & - & - \\
\hline $6 b$ & 1000 & - & - & - & 10 & - & - & - & - & 11 & - & - & - \\
\hline $7 a$ & 1000 & - & - & - & 12 & - & - & - & - & - & - & - & - \\
\hline $8 \mathbf{a}$ & 1000 & - & - & - & 12 & - & - & - & - & - & - & - & - \\
\hline $8 b$ & 1000 & - & - & - & - & - & - & 13 & - & 11 & - & - & - \\
\hline $9 a$ & 1000 & - & - & - & 12 & - & - & 12 & - & - & - & - & - \\
\hline $9 b$ & 1000 & - & - & - & - & - & - & 14 & - & - & - & - & - \\
\hline $10 a$ & 1000 & - & - & - & 10 & - & - & - & - & 11 & - & - & - \\
\hline $10 \mathrm{~b}$ & 1000 & - & - & - & - & - & - & 11 & - & - & - & - & - \\
\hline Ampicillin & & & 8 & 5 & 5 & 5 & 11 & 15 & 14 & & & - & - \\
\hline Fortum & & & 45 & 45 & 45 & 20 & 30 & 30 & 35 & & & & \\
\hline Triflucan & & & & & & & & & & 25 & 25 & & \\
\hline
\end{tabular}

Ec; Escherichia coli ATCC 25922, Sa; Staphylococcus aureus ATCC 25923,Pa; Pseudomonas aeruginosa ATCC 27853, Ah; Acinetobacter haemolyticus ATCC 19002; Bs; Bacillus subtilis ATCC 6633, Pv; Proteus vulgaris ATCC 13315, Entc; Enterobacter cloacea ATCC 13047, Ef; Enterococcus faecalis ATCC 29212, Ca; Candida albicans ATCC 60193, Af; Aspergillus sp., Fusa; Fusarium sp., Rhyso; Rhizopus sp. 


\section{Conclusions}

In this study, a series of new triazole derivatives having carbohydrazide, thiosemicarbazide, thiadiazole and triazole-thiol moieties, respectively, at the 1-position was synthesized, and their antioxidant and antimicrobial activities were evaluated. It was observed that in vitro the newly synthesized triazole-thiosemicarbazides $\mathbf{4 a}, \mathbf{b}-\mathbf{6} \mathbf{a}, \mathbf{b}$ possess highly potent antioxidant properties and triazole/triazole-thiol derivatives $\mathbf{1 0 a}, \mathbf{b}-\mathbf{1 1 a}, \mathbf{b}$ possess moderate potent antioxidant properties. Compounds $\mathbf{7 a}, \mathbf{b}, \mathbf{8 a}, \mathbf{b}$ and $\mathbf{9 a}, \mathbf{b}$, containing triazole-thiadiazole and triazole/triazole-thiol moieties didn't show antioxidant properties. All newly synthesized compounds were screened for their antibacterial and antifungal activities by the inhibition zones ( $\mathrm{mm}$ ) method. Almost all the synthesized compounds showed significant activity against bacteria, while no compounds showed activity against fungi (Aspergillus, Fusarium, Rhizopus). In particular the 1,2,4-triazole-possessing thiosemicarbazides $\mathbf{4 a , b}-\mathbf{6 a}, \mathbf{b}$ show both antibacterial and antifungal activities.

\section{Acknowledgments}

This work was supported by the Research Fund of Karadeniz Technical University. The authors thank Atalay Sökmen for the antioxidant and antimicrobial studies.

\section{Conflicts of Interest}

The authors declare no conflict of interest.

\section{References}

1. Unangst, P.C.; Shrum, G.P.; Dyer, D.T.; Schrier, D.J. Novel 1,2,4-oxadiazoles and 1,2,4thiadiazoles as dual 5-lipoxygenase and cyclooxygenase inhibitors. J. Med. Chem. 1992, 35, 3691-3698.

2. Mullican, M.D.; Wilson, M.W.; Connor, D.T.; Kostlan, C.R.; Schrier, D.J. Design of 5-(3,5-ditert-butyl-4-hydroxyphenyl)-1,3,4-thiadiazoles, -1,3,4-oxadiazoles, and -1,2,4-triazoles as orally active, nonulcerogenic antiinflammatory agents. J. Med. Chem. 1993, 36, 1090-1099.

3. Boschelli, D.H.; Conner, D.T.; Bornemeir, D.A.; Dyer, R.D.; Kennedy, J.A.; Kuipers, P.J.; Okonkwo, G.C.; Schrier, D.J.; Wright, C.D. 1,3,4-oxadiazole, 1,3,4-thiadiazole, and 1,2,4-triazole analogs of the fenamates: In vitro inhibition of cyclooxygenase and 5-lipoxygenase activities. J. Med. Chem. 1993, 36, 1802-1810.

4. Jones, D.H.; Slack, R.; Squires, S.; Wooldridge, K.H. Antiviral chemotherapy. I. The activity of pyridine and quinoline derivatives against neurovaccinia in mice. J. Med. Chem. 1965, 8, 676-680.

5. Kelarev, V.I.; Karakhanov, R.A.; Gasanvo, S.; Morozova, G.V.; Kuatbekova, K.P. Synthesis of 2-amino-sym-triazines containing alkyl radicals. Chem. Heterocycl. Compd. 1993, 29, 1087-1092.

6. Habib, N.S.; Abdel-Hamid, S.; El-Hawash, M. Synthesis of benzimidazole derivatives as potential antimicrobial agents. Il Farmaco 1989, 44, 1225-1232.

7. Stillings, M.R.; Welbour, A.P.; Walter, D.S. Substituted 1,3,4-thiadiazoles with anticonvulsant activity. J. Med. Chem. 1986, 29, 2280-2284. 
8. Kane, J.M.; Staeger, M.A.; Dalton, C.R.; Miller, F.P.; Dubley, M.W.; Ogden, A.M.L.; Kehne, J.H.; Ketteler, H.J.; McCloskey, T.C.; Senyah, Y. 5-Aryl-3-(alkylthio)-4H-1,2,4-triazoles as selective antagonists of strychnine-induced convulsions and potential antispastic agents. J. Med. Chem. 1994, 37, 125-132.

9. Chapleo, C.B.; Myers, M.; Myers, P.L.; Saville, J.F.; Smith, A.C.B.; Stillings, M.R.; Tulloch, I.F.; Walter, D.S.; Welbour, A.P. Substituted 1,3,4-thiadiazoles with anticonvulsant activity. J. Med. Chem. 1986, 29, 2273-2280.

10. Kane, J.M.; Dubley, M.W.; Sorenson, S.M.; Miller, F.P. 2,4-Dihydro-3H-1,2,4-triazole-3-thiones as potential antidepressant agents. J. Med. Chem. 1988, 31, 1253-1258.

11. Mano, M.; Matsuno, T.; Imai, K. Anticoccidials I. Syntheses and anticoccidial activity of 2-amino-5-aryl-1,3,4-oxadiazoles, 5-alkoxy-3-aryl-1H-1,2,4-triazoles, and 3-aryl-1,2,4-triazolin-5ones. Chem. Pharm. Bull. 1976, 24, 2871-2876.

12. Gürsoy, A.; Demirayak, S.; Cesur, Z.; Reisch, J.; Otük, G. Synthesis of some new hydrazidehydrazones, thiosemicarbazides, thiadiazoles, triazoles and their derivatives as possible antimicrobials. Pharmazie 1990, 45, 246-250.

13. Hiremath, S.P.; Biradar, J.S.; Kuradi, S.M. Synthesis of substituted oxadiazoles, thiadiazoles and triazoles and their biologocal activity. J. Indian Chem. Soc. 1984, 61, 74-76.

14. Amir, M.; Kumar, H.; Javed, S.A. Condensed bridgehead nitrogen heterocyclic system: Synthesis and pharmacological activities of 1,2,4-triazolo-[3,4]-1,3,4-thiadiazole derivatives of ibuprofen and biphenyl-4-yloxy acetic acid. Eur. J. Med. Chem. 2008, 43, 2056-2066.

15. Navidpour, L.; Shafaroodi, H.; Abdi, K.; Amini, M.; Ghahremani, M.H.; Dehpourd, A.R.; Shafiee, A. Design, synthesis, and biological evaluation of substituted 3-alkylthio-4,5-diaryl-4H1,2,4-triazoles as selective COX-2 inhibitors. Bioorg. Med. Chem. 2006, 14, 2507-2517.

16. Cukurovali, A.; Yilmaz, I.; Gur, S.; Kazaz, C. Synthesis, Antibacterial and Antifungal Activity of Some New Thiazolylhydrazone Derivatives Containing 3-Substituted Cyclobutane Ring. Eur. J. Med. Chem. 2006, 41, 201-207.

17. Tehranchian, S.; Akbarzadeh, T.; Fazeli, M.R.; Jamalifar, H.; Shafiee, A. Synthesis and antibacterial activity of 1-[1,2,4-triazol-3-yl] and 1-[1,3,4-thiadiazol-2-yl]-3-methylthio-6,7dihydrobenzo[c]thiophen-4(5H)ones. Bioorg. Med. Chem. Lett. 2005, 15, 1023-1025.

18. Holla, B.S.; Poojary, K.N.; Rao, B.S.; Shivananda, M.K. New bis-aminomercaptotriazoles and bis-triazolothiadiazoles as possible anticancer agents. Eur. J. Med. Chem. 2002, 37, 511-517.

19. Ghorab, M.M.; El-Sharief, A.M.S.; Ammar, Y.A.; Mohamed, S.I. Synthesis and radiation stability of novel biologically active sulfur compounds derived from 1,2-bis(4-amino-5-mercapto-s-triazol3-yl)ethane. Il Farmaco 2000, 55, 354-361.

20. İkizler, A.A.; Sancak, K. Synthesis of 4-Hydroxy-4,5-dihydro-1,2,4-triazol-5-ones. Collect. Czech. Commun. 1995, 60, 903-909.

21. Burits, M.; Bucar, F. Antioxidant activity of Nigella sativa essential oil. Phytother. Res. 2000, 14, 323-328.

22. Cuendet, M.; Hostettmann, K.; Potterat, O. Iridoid glucosides with free radical scavenging properties from Fagraea blumei. Helv. Chim. Acta 1997, 80, 1144-1152. 
23. Finegold, S.M.; Martin, W.J. Bailey and Scott's Diagnostic Microbiology, 6th ed.; C.V. Mosby Co.: St. Louis, MO, USA, 1982.

Sample Availability: Not available.

(C) 2014 by the authors; licensee MDPI, Basel, Switzerland. This article is an open access article distributed under the terms and conditions of the Creative Commons Attribution license (http://creativecommons.org/licenses/by/3.0/). 